\section{Lesiones orales como factores de riesgo de enfermedades sistémicas}

\author{
Oral lesions as Risk Factors of Systemic Diseases
}

Artículo DE REVISIÓN

Luis Cuadrao Zavaleta ${ }^{1}$, Ricardo M. Romero Márquez ${ }^{2}$

1. Departamento Académico de Ciencias Básicas. Facultad de Odontología. Universidad Nacional Mayor de San Marcos. Perú.

Los problemas en la salud oral influyen en la condición general del individuo. El diagnóstico clínico de la enfermedad oral puede dar idea de la causa y el pronóstico, sin embargo no refleja directamente el nivel de alteración en el individuo. En algunas personas las enfermedades orales indican un factor de riesgo pero que no son de por vida, ni tan severos que deshabiliten sus capacidades, sin embargo sus consecuencias pueden impactar en la vida diaria en diferentes grados y provocar la percepción de incapacidad alterando el desenvolvimiento psicosocial. Diversos autores mencionan signos orales como factor de riesgo de enfermedades sistémicas. Patologias del canal radicular como lugar de brote de actinomicosis sistémica. Tambien se describe a la ulceración crónica del paladar duro donde se presenta el signo de tuberculosis pulmonar no diagnosticada, asi mismo la sífilis secundaria es inicialmente diagnosticada por lesiones orales. Otros autores sost ienen que el Sarcoma de Ewing primario envuelve al hueso de la cabeza y el cuello antes de repercutir sistemicamente. Tambien se describe que el Citomegalovirus en mucosal oral es una de las primeras manifestaciones del SIDA. Asi mismo se dice que la exposición hematógena a los estreptococos orales ha sido reconocido como potencial factor iniciador de la endocarditis bacteriana. La infección ha sido reconocida como factor de riesgo para la aterogénesis y los eventos trombóticos. Se encontró asociación significativa entre el componente periodontal del índice dental y la isquemia cerebrovascular. Hay lesiones orales que son factores de riesgo para desarrrollar una enfermedad sistémica. La osteodistrofia renal y los cambios en el tejido oseo son consecuencia de la insuficiencia renal. Los cambios en el hueso alveolar asociados a la osteodistrofia renal son similares a los hallados en el hiperparatiroidismo primario e incluyen pérdida generalizada de densidad ósea, adelgazamiento de la cortical de los huesos y pérdida total o parcial de la lámina dura.

Palabras clave: cavidad oral, factor de riesgo, enfermedades sistémicas.

\begin{abstract}
Oral health problems influence the general condition of people. The clinical diagnosis of oral disease can give an idea of the etiology and prognosis, but this not directly reflects the level of disturbance. For some people oral diseases indicate a risk factor but are not for life, not severe enough to disable their abilities, but their consequences can impact daily life at different degrees and cause disability altering the perception of psychosocial development. Several authors mention oral signs as risk factor for systemic diseases. Pathologies of root canal considered as a place of outbreak of systemic actinomycosis. Chronic ulceration of the hard palate where the sign of undiagnosed pulmonary tuberculosis is presented is also described; likewise secondary syphilis is initially diagnosed by oral lesions. Other authors argue that primary Ewing sarcoma of bone surrounds the head and neck before impact systemically. It is also described the presence of cytomegalovirus in oral mucosal as one of the first manifestations of AIDS. Hematogenous exposure to oral streptococci has been recognized as potential initiator factor of bacterial endocarditis. The infection has been recognized as a risk factor for atherogenesis and thrombotic events. Significant association between periodontal component of the dental index and cerebrovascular ischemia were found. There are oral lesions that are risk factors for developing systemic disease. Renal osteodystrophy and changes in the bone tissue are a consequence of renal failure. Changes in alveolar bone associated with renal osteodystrophy are similar to those found in primary hyperparathyroidism and include generalized loss of bone density, thinning of the cortical bone and partial or total loss of the lamina dura.
\end{abstract}

Keywords: oral cavity, risk factors, systemic diseases.

\section{Introduccion}

La evaluación de las lesiones orales para medir la calidad de vida relacionada a la salud general tiene por objetivo evaluar no sólo la función física y el dolor, sino también los constructores psicosociales y la satisfacción de vivir. Uno de los modelos teóricos propuesto por Locker $^{1}$ busca relacionar las variables biológicas de la enfermedad con la percepción de la persona acerca del impacto de estas en su vida. Se han empleado ampliamente estas medidas en el campo del cáncer oral, síndrome de Sjogren, en la población general, en los ancianos y en los edéntulos, pero muy poco se han utilizado para evaluar el impacto de los problemas de salud oral en individuos referidos a hospitales para tratamientos por desordenes estomatológicos: como infecciones virales o bacterianas, candidiasis o liquen plano los cuales provocan dificultades al comer o hablar; también desórdenes funcionales como boca seca, condiciones dolorosas en una neuralgia
Fecha de recepción: 05-05-13

Fecha de aceptación. 19-06-14

o disfunción de la articulación temporo mandibular, alteraciones de las glándulas salivales o a nivel óseo. La evaluación de estas lesiones bucales son un puente de unión entre la decisión clínica de las estrategias de manejo de la enfermedad por el clínico y el paciente. Muchas veces el tratamiento establecido en medicina oral está en contra de la experiencia del paciente, es decir, no está relacionado con la severidad de la enfermedad. Así mismo es importante para la evaluación longitudinal del tratamiento en 
el paciente. Existe una evidente relación entre las lesiones orales con la ansiedad y la depresión en individuos con tratamientos de por vida como cáncer de cabeza y cuello.

\section{Manifestaciones Orales}

Indudablemente los problemas en la salud oral influyen en la condición general de la persona. El diagnóstico clínico de la enfermedad oral puede dar idea de la causa y el pronóstico, sin embargo no refleja directamente el nivel de alteración en el individuo. Para la mayoría de las personas las enfermedades orales no son de por vida, ni tan severos que deshabiliten, sin embargo sus consecuencias pueden impactar en la vida diaria en diferentes grados y provocar la percepción de incapacidad alterando el desenvolvimiento psicosocial ${ }^{1}$. Se menciona el canal radicular como lugar de brote de actinomicosis sistémica ${ }^{2}$. Tambien se describe a la ulceración crónica del paladar duro como el primer signo de tuberculosis pulmonar no diagnosticada $^{3}$. La sífilis secundaria es inicialmente diagnosticada por lesiones orales ${ }^{4}$. El Sarcoma de Ewing primario envuelve al hueso de la cabeza y el cuello antes de repercutir sistemicamente . $^{5}$ El citomegalovirus de mucosa oral es una de las primeras manifestaciones del SIDA ${ }^{6}$. la sistémica exposición hematógena a los estreptococos orales ha sido reconocido como potencial factor iniciador de la endocarditis bacteriana ${ }^{7}$. La infección tambien reconocida como factor de riesgo para la aterogenesis y los eventos trombóticos ${ }^{7}$. En otra investigacion se encontró asociación significativa entre el componente periodontal del índice dental y la isquemia cerebrovascular ${ }^{8}$.

Heimdahl y col (9) en 1989 indican que la cavidad oral es una puerta de entrada para las infecciones tempranas en pacientes tratados con trasplante medular óseo. También la relaciona con los pacientes con otras enfermedades sistémicas.

\section{Relación entre enfermedad periodon-} tal y enfermedad cardiovascular

Varios estudios de casos y controles longitudinales muestran relación entre las enfermedades periodontales y las enfermedades cardiovasculares entre débil a moderada, en diversas poblaciones parece ser independiente de los riesgos tradicionales. La infección periodontal puede directamente contribuir a la ateroesclerosis y la trom- boembolia al proveer cambios repetidos en el sistema vascular por efecto de los lipopolisacáridos, las citoquinas de la inflamación y las bacterias mismas ${ }^{7}$. El significado potencial de las bacterias orales Gram positivas, incluyendo las especies estreptocócicas, como estreptococos sanguis han sido investigados, donde la exposición hematógena sistémica a los estreptococos orales se han reconocido como potencial factor iniciador de la endocarditis bacteriana? Algunos de los experimentos iniciales de inmunización con estreptococos contra la caries dental han producido anticuerpos anti vasculares y anti cardiacos en reacciones inmunológicas cruzadas ${ }^{7}$. El estreptococos sanguis posee una proteína externa de membrana con 7 secuencias ProGlyGluGinGlyPro-Lys al dominio plaquetario del colágeno tipo I y II, estas proteínas bacterianas simulan los receptores plaquetarios normales que inician la formación de trombos ${ }^{7}$. También reportaron la Porphyromonas gingivalis, microorganismo periodontopatógeno Gram negativo, con similares propiedades ${ }^{7}$. Así mismo se reportó que 19 de 27 ateromas obtenidas de pacientes durante endarterectomias fueron positivos para DNA bacterial de patógenos periodontales. De los 19, 6 fueron positivos para el actinobacillus actinomycetemcomitans, 6 para P. gingivalis y 7 para prevotella intermedia ${ }^{7}$.

La infección periodontal reconocida como factor de riesgo para la aterogénesis y los eventos trombóticos ${ }^{7}$. Las bacterias Gram negativas o las endotoxinas lipopolisacáridos asociados, son un desafío sistémico en los modelos animales puede producir infiltración de células inflamatorias en los vasos sanguíneos grandes, degeneración grasa de los vasos y coagulación intravascular ${ }^{7}$. La similitud remarcable entre la patología vascular inducida por bacterias y la historia natural de la ateroesclerosis ha conllevado a algunos investigadores a sugerir que aparte de las influencias genéticas, el estilo de vida, los factores dietéticos, las infecciones de origen desconocido pueden contribuir a la patología cardiovascular ${ }^{7}$. Las reacciones a la inflamación crónica en la infección periodontal proveen la base para el modelo hipotético de las relaciones observadas entre la aterosclerosis y la enfermedad periodontal ${ }^{7}$. La ateroesclerosis, definida como una enfermedad progresiva que implica el grosor de arterias musculares de mediano calibre y las grandes arterias elásticas, cuya complicación frecuente es infarto miocárdico o cerebral tiene factores en común con la enfermedad periodontal: ocurren con mayor frecuencia en perso- nas adultas, varones, de bajo nivel educacional, con pocos recursos financieros, que fuman, son estresados y socialmente aislados ${ }^{6}$.

Un grupo de ocho estudios (tres de casos control y cinco prospectivos) son presentados donde se comparan 100 pacientes con infarto agudo de miocardio con 102 controles de la comunidad seleccionados al azar, pareados por edad, sexo y vecindad ${ }^{12}$. El examen dental implicó la evaluación del índice de caries dental, la condición periodontal: profundidad de bolsas (incluyendo presencia de pus en estos), el número de lesiones peri apicales y la presencia o ausencia de pericoronitis (Índice dental total) ${ }^{12}$. Se mostró que los pacientes tenían peor salud dental que los controles. El análisis de regresión indicó asociación entre la pobre salud oral y la enfermedad coronaria persistente en los controles para edad, colesterol total, lipoproteínas de alta densidad, triglicéridos, péptidos $\mathrm{C}$, hipertensión, presencia de diabetes y hábito de fumar ${ }^{12}$.

También utilizando el índice de infección dental total ${ }^{10}$. Encontraron asociación significativa entre el componente periodontal del índice dental y la isquemia cerebrovascular ${ }^{8}$.

En un estudio prospectivo de 7 ańos ${ }^{10}$, incluyeron pacientes que habían tenido experiencia de infarto de miocardio para determinar la ocurrencia de eventos coronarios fatales o no y la mortalidad promedio ${ }^{10}$. La evaluación dental incluyó el Índice dental total IDT (caries, periodontitis, lesiones peri apicales, peri coronitis) y el índice el pantomográfico IP (número de bolsas verticales, furcaciones, lesiones peri apicales). El IDT y el pantomográfico fueron significativos para la ocurrencia de nuevos eventos coronarios en un modelo donde se incluía número de infartos previos, diabetes, índice de masa corporal, hipertensión, hábito de fumar, colesterol total, colesterol de lipoproteínas de alta densidad, triglicéridos, estatus socioeconómico, edad y sexo ${ }^{10}$.

De Stefano et al (11) investigaron la enfermedad coronaria y su mortalidad por 14 años, demostrando que en alrededor de los 10,000 sujetos analizados, aquellos con periodontitis tenían incremento de riesgo de enfermedad coronaria en $25 \%$ en comparación a aquellos con mínimas alteraciones periodontales, ajustando el análisis para las variables de confusión potencial como: edad, sexo, raza, educación, estado marital, presión sanguínea sistólica, niveles de colesterol 
total, índice de masa corporal, diabetes, actividad física, alcohol, pobreza y hábito de fumar. En hombres menores de 52 ańos la enfermedad periodontal tuvo efecto de incidencia sobre la enfermedad coronaria con un factor de riesgo de 1.72

Otros estudios reportaron asociación entre el nivel de pérdida ósea por enfermedad periodontal y la enfermedad cardiovascular así como asociación entre la infección dental y factores séricos: factor VIII, actividad del cofactor ritcocetin del factor VIII y el factor de Von Willebrand ${ }^{12131415}$.

También otro estudio comparó el fibrinógeno y el recuento de células blancas en sangre venosa entre 50 pacientes consecutivos con gingivitis o periodontitis entre 25 y 50 años de edad y controles periodontalmente sanos ${ }^{16}{ }^{17}$. Donde evaluaron el índice de placa, índice gingival y el índice periodontal de necesidades de tratamiento. Mostrando que los pacientes periodontales tenían significativamente más alto el nivel de fibrinógeno y el recuento de células blancas, observaron asociación entre el índice gingival y en ambos parámetros aumento de fibrinógeno y células blancas. Cada una de estas asociaciones fue independiente para edad, hábito de fumar, estatus social y estatus grupal ${ }^{16}{ }^{17}$.

\section{Disfunción renal y salud periodontal}

La osteodistrofia renal y los cambios en el tejido óseo relacionados son consecuencias de la insuficiencia renal. Clínicamente, los niños con esta enfermedad pueden experimentar retardo del crecimiento y fracturas óseas frecuentes. En adultos, la presentación clínica incluye curvaturas y fracturas de los huesos largos debidas al ablandamiento gradual de los huesos con el tiempo ${ }^{18}$.

El mecanismo subyacente a la osteodistrofia renal es un defecto en la hidroxilación del precursor de la vitamina D, la 25-hidroxivitamina D (calcidiol o hidrocolecalciferol) a 1,25-dihidroxivitamina D (calcitriol o dihicrocolecalciferol), un proceso que ocurre normalmente en el riñón. Dado que una función primaria de la 1,25-dihidroxivitamina $\mathrm{D}$ es estimular la absorción del calcio por el intestino, los individuos con este trastorno experimentan con frecuencia hipocalcemia. El estado hipocalcémico resulta en la secreción de hormona paratiroidea ${ }^{18}$. El aumento de la hormona paratiroidea es secundario al desequilibrio en los niveles de calcio $y$ fósforo, por lo que recibe el nombre de hiperparatiroidismo secundario. El efecto neto de este desequilibrio es la reabsorción ósea, al intentar el organismo mantener el equilibrio homeostático. Los cambios en el hueso alveolar asociados a la osteodistrofia renal son similares a los hallados en el hiperparatiroidismo primario e incluyen pérdida generalizada de densidad ósea, adelgazamiento de la cortical de los huesos y pérdida total o parcial de la lámina dura $^{18}$. Los individuos con osteodistrofia pueden presentar también una trabeculación disminuida, con aumento concomitante del espacio medular. Esta pérdida de trabeculación puede continuar hasta que el hueso ofrece una apariencia homogénea, a menudo descrita como «vidrio molido» o «tiza».

Otras zonas pueden presentar un aumento de densidad (osteosclerosis). Aunque las lesiones de osteosclerosis están bien documentadas ${ }^{18}$, actualmente no existe un mecanismo biológico demostrado que las explique. Los desequilibrios fisiológicos y los cambios óseos resultantes provocados por la insuficiencia renal crónica a menudo se reducen o revierten cuando se trata la enfermedad renal subyacente, ya sea mediante diálisis renal o trasplante de riñón ${ }^{19}$. No se sabe si también podría darse la reversibilidad de los cambios en el hueso oral. Los pacientes en diálisis renal parecen tener una prevalencia mayor de periodontitis ${ }^{19}$. Es probable que la disminución de densidad mineral ósea en tales pacientes aumente el riesgo de pérdida progresiva de hueso alveolar debida a las infecciones periodontales subsiguientes, de forma similar a lo que se cree que ocurre en pacientes con osteoporosis ${ }^{18}{ }^{19}$. Además, cuando se prepara a estos individuos para trasplante de órganos, es importante que se les haga una exploración dental cuidadosa, y que cualquier fuente de infección dental se elimine antes del trasplante, ya que las complicaciones de las infecciones pueden ser graves y llevar a una morbilidad significativa, incluyendo la insuficiencia del órgano trasplantado ${ }^{19}$

\section{Discusión}

Las diversas enfermedades que se mencionaron son una fuente de factores que pueden determinar o aumentar los riesgos de enfermedades sistémicas.

Se demuestra la relación entre la enfermedad periodontal y las enfermedades cardiovasculares, así como la asociación entre periodontitis, enfermedad cardiovascular, marcadores hematológicos y rheológicos.

\section{Conclusiones}

En nuestro país hay muchas lesiones orales que repercuten sistémicamente sin ser reportado o confirmado su diagnóstico para su prevención y tratamiento.

Existe la necesidad de actualizar los conocimientos de las lesiones orales y su repercusión sistémica.

La presente revisión bibliográfica buscó actualizar los conocimientos de las lesiones orales y sus relaciones con las diferentes enfermedades sistémicas, las cuales no están muy difundidas entre los otros miembros del equipo de salud y que son muy importantes y determinantes en la etiología, pronóstico, diagnóstico y tratamiento de las enfermedades sistémicas con manifestaciones orales iniciales.

\section{Referencias bibliográficas}

1. Carranza F. Periodontología Clínica. 9 ed. México: McGraw-Hill Interamericana; 2004. pp.138-62.

2. Borssen $\mathrm{E}$ et al. Actinomyces of infected dental root canals. Oral Surg Oral Med Oral Pathol. 1981 Jun;51(6):6438.

3. Michaud M. y col. Cronic ulceration of the hard palate: first clinical sign of the undiagnosed pulmonary tuberculosis. Oral Surg Oral Med Oral Pathol. 1984 Jan;57(1):63-7.

4. Mani N. Secondary syphilis initially diagnosed from oral lesion. Oral Surg Oral Med Oral Pathol. 1984;58(1):4750

5. 5. Siegal G. et al. Primary Ewing Sarcoma involving the bones of the head and neck. Cáncer 1987;60: 2829-2840

6. Kanas $\mathrm{R}$ et al. Oral mucosal cytomegalovirus as a manifestation of the acquired mmunodeficiency syndrome. Oral Surg Oral Med Oral Pathol. 1987;64(2):183-9.

7. Hertzberg M, MacFarlane G, Liu P, Erickson P. The platelet as an antiinflammatory cell in periodontal diseases: interaction with Porphyromonas gingivalis. Genco RAm SoeMicrobiol 1994: 247-255.

8. Grau A., Buggle E, Siegler C. Association between cerebrovascular ischemia and chronic and recurrent infection. Stroke 1997:28:1724-1729.

9. Heinmdahl y col. The oral cavity as a part of entry for early infections in patients treated with bone marrow trans- 
plantation. Oral Surg Oral Med Oral Pathol. 1989;68(6):711-6.

10. Mattila K., Nieminen M., Valtonen V et al. Association between dental health and acute myocardial infarction. $\mathrm{Br}$ Med J. 1989 Mar 25:298(6676):77981.

11. DeStefano E, Anda RE, Kahn HS, Williamson DF, Russel CM. Dental disease and risk of coronary heart disease and mortality. BMJ 1993:306:688-691.

12. Joshipura KJ, Rimm EB, Douglass CW, Trichopoulos D, Ascherio A, Willett WC.Poor oral health and coronary heart disease. J Dent Res. 1996:75:1631-1636.
13. Beck JD, Slade G, Offenbaches S. Oral disease, cardiovascular disease and systemic inflammation. Periodontology 2000. 2000:23:110-120.

14. Genco RJ. Periodontal disease is a predictor of cardiovascular disease in a $\mathrm{Na}$ tive American population. [abstract]. J Dent Res. 1997;76 (special issue):3158.

15. Mattila K, Rasi V, Nieminen M. et al. Von Willebrand factor antigen and dental infections. Thromb Res. 1989;56:325-329.

16. Kweider M, Lowe GD, Murray GD, Kinane DF, McGowan DA. Dental disease, fibrinogen and white cell count; links with myocardial infarction?. Scott Med J. 1993;38:73-74.
17. Ebersole J, Machen R, Steffen M, Willmann D. Systemic acutephase reactant, C-reactive protein and haptoglobin in adult periodontitis. Clin Exp Immunol 1997:107:347-352.

18. Kelly WH, Mirahmadi MK, Simon JH, Gorman JT. Radiographic changes of the jawbones in end stage renal disease. Oral Surg Oral Med Oral Pathol. 1980 Oct;50(4):37281 .

19. Julian BA, Laskow DA, Dubovsky J, Dubovsky EV, Curtis JJ, Quarles LD. Rapid loss of vertebral mineral density after renal transplantation. N Engl J Med. 1991 Aug 22;325(8):544-50. 